\title{
RÉPUBLIQUE ET DÉMOCRATIE DE MONTESQUIEU À MADISON
}

\author{
Jean-Fabien SPITZ
}

\begin{abstract}
Résumé : La théorie politique est longtemps restée attachée au dogme selon lequel la république ne peut convenir qu'à de petits États, où la faible différenciation des conditions sociales autorise la formation d'un authentique intérêt commun. Montesquieu adopte une version de cet argument en soutenant que la politique moderne doit être non la recherche de l'intérêt commun, mais l'art de balancer les intérêts les uns contre les autres. Madison a répondu en montrant comment, au contraire, la république n'est possible que dans les grands États, où la diversité des intérêts en présence les contraint à rechercher non pas un compromis - impossible à maintenir quand il faut unir un grand nombre d'intérêts distincts pour former une majorité mais un dépassement de leurs particularités respectives au profit d'un authentique intérêt commun.
\end{abstract}

MoTs-CLÉs : démocratie, république, constitution, factions, liberté.

ABSTRACT: Political theory has been for long committed to the idea that a republic is possible only in small states, where the diversity of interests is reduced to the point where it makes possible the formation of a genuine common interest. Montesquieu adheres to this thesis when he says that, far from looking for this chimerical common interest, modern politics must be an art of balancing particular interests. Madison answered this argument by showing how, on the contrary, a republic is possible in large states only; in such states, the variety of extant interests constrains each of them to look not for a compromise - which is impossible to find where the interests in play are so widely apart from each other - but for a genuine common interest transcending all particularities.

KEYWORDS : democracy, republic, constitution, factions, political freedom.

Revue de synthèse : $4^{e} \mathrm{~S} . \mathrm{N}^{\mathrm{os}}$ 2-3, avril-septembre 1997, p. 259-283. 
ZuSAMMENFASSUNG: Die politische Theorie hat lange an der Vorstellung festgehalten, daß die republikanische Staatsform nur in kleinen Staaten möglich ist, in denen eine geringe Differenzierung der sozialen Bedingungen die Entstehung eines genuinen gemeinsamen Interesses möglich macht. Auch Montesquieu macht sich dieses Argument zueigen, wenn er erklärt, es sei nicht Aufgabe der modernen Politik, danach zu fragen, wie jenes angebliche gemeinsame Interesse beschaffen ist, sondern sie müsse darauf bedacht sein, zwischen den verschiedenen Interessen auszugleichen. Madison führte dagegen den Nachweis, daß die republikanische Staatsform nur in großen Staaten möglich ist. In solchen Staaten gehen die einzelnen Interessen so weit auseinander, daß es unmöglich ist, Kompromisse zu finden. Statt dessen ist die Politik dort genötigt, ein gemeinsames Interesse zu finden, das über alle Partikularinteressen hinausgeht.

STICHWÖRTER : Demokratie, Republik, Verfassung, Parteien, Freiheit.

Jean-Fabien SPrrz, né en 1952, ancien élève de l'École normale supérieure, enseigne la philosophie politique à l'université de Caen. Il a récemment publié La Liberté politique. Essai de généalogie conceptuelle, Paris, Presses universitaires de France («Léviathan »), 1995.

Adresse: Département de philosophie, Université de Caen, Esplanade de la Paix, 14000 Caen. 
Le républicanisme contemporain peut être un outil théorique pour lutter contre certaines représentations politiques aujourd'hui puissantes mais pernicieuses. L'une d'entre elles est le libertarisme, qui a été critiqué de manière pertinente par Quentin Skinner et Philip Pettit '. Nous voudrions montrer ici comment une autre doctrine très souvent évoquée dans le débat contemporain - la théorie «élitiste » de la démocratie - pourrait être utilement mise en perspective par les principes d'une philosophie républicaine.

La théorie élitiste ${ }^{2}$ soutient que l'existence du gouvernement du peuple par le peuple et pour le peuple - la démocratie en son sens originel - est une chimère qui débouche nécessairement soit sur la tyrannie de la majorité soit sur l'anarchie et, à terme, sur la tyrannie d'un seul; elle tend à présenter le gouvernement du peuple par le peuple comme incompatible avec l'existence de droits individuels, et elle prétend montrer que la démocratie est toujours l'ennemie de la liberté; elle s'appuie sur l'idée - dont l'origine remonte à Hobbes - que les droits de l'individu sont aussi peu à l'abri des décisions de la majorité sous le pouvoir souverain d'un peuple, que des caprices de la fantaisie du prince sous le gouvernement d'un seul.

Le caractère pernicieux de cette théorie est qu'elle contribue à autonomiser les élites dirigeantes, comme si celles-ci possédaient un intérêt qui devrait avoir en quelque sorte un poids spécifique à l'intérieur de l'État, et dont la présence et l'activité seraient utiles et même indispensables pour équilibrer le poids de l'élément démocratique. Une telle théorie affirme donc que, lorsqu'elle n'est pas corrigée par des mécanismes élitaires, la démocratie tend à l'excès, à l'inquisition et à la tyrannie. Or, cette indépendance relative des élites dans le fonctionnement de l'État est un facteur indéniable de corruption. À son tour, la corruption menace les fondements de la liberté, puisque celle-ci repose sur la réciprocité absolue des droits et des devoirs entre les magistrats et les citoyens ${ }^{3}$.

Le républicanisme peut nous apprendre à réfuter ces théories élitistes de la démocratie en montrant que le gouvernement par le peuple n'a pas, à condition qu'on sache comment l'organiser, les conséquences qu'elles

1. Quentin SkINNER, "The republican idea of political liberty », in Machiavelli and republicanism, éd. Gisela Bock, Quentin SkinNer, Maurizio Virol, Cambridge, Cambridge University Press, 1990; cf. Philip PETrit, "Liberalism and republicanism ", Australian Jourmal of political science, $\mathrm{n}^{\circ}$ spécial, vol. 28, 1993, p. 162-189.

2. Cf. Moses I. Fintey, Démocratie antique et démocratie moderme, Paris, Payot, 1976; Bernard Manis, Principes du gouvernement représentatif, Paris, Calmann-Lévy, 1995, p. 208 sq.

3. Cf. P. PEtTit, art. cit. supra n. 1. 
dénoncent : la république serait alors considérée comme l'art de combiner le principe de la démocratie avec celui de la liberté, le principe de la souveraineté sans partage du peuple avec la maîtrise des effets de cet esprit de faction qui peut mener à la tyrannie de la majorité. Elle nous apprend par conséquent à couper court à tout raisonnement qui tendrait à montrer que la corruption est, sinon légitime, du moins inévitable dans un régime qui ne veut pas tomber dans le caractère inquisitorial qui caractérise tous les États où la majorité et la volonté populaire ne rencontrent aucun obstacle dans l'exécution de leurs décisions.

MONTESQUIEU : LE DANGER DE LA DÉMOCRATIE DANS LES GRANDS ÉTATS

Dans L'Esprit des lois, Montesquieu a donné une formulation classique de la raison pour laquelle les grands États modernes ne pouvaient pas vivre sous le régime de la démocratie pure ou directe (qu'il appelle le gouvernement républicain). Ce gouvernement, dit-il, ne convient qu'à des États de petites dimensions :

«Il est de la nature d'une république qu'elle n'ait qu'un petit territoire : sans cela, elle ne peut guère subsister. Dans une grande république, il y a de grandes fortunes, et par conséquent peu de modération dans les esprits; il y a de trop grands dépôts à mettre entre les mains d'un citoyen; les intérêts se particularisent; un homme sent d'abord qu'il peut être heureux, grand, glorieux sans sa patrie; et bientôt, qu'il peut être seul grand sur les ruines de sa patrie.

Dans une grande république, le bien commun est sacrifié à mille considérations; il est subordonné à des exceptions; il dépend des accidents. Dans une petite, le bien public est mieux senti, mieux connu, plus près de chaque citoyen; les abus y sont moins étendus et par conséquent moins protégés ${ }^{4}$.»

Les grandes fortunes induisent en effet ceux qui les détiennent à penser que leurs intérêts pourraient être satisfaits non par une fusion avec l'intérêt des autres dans l'intérêt commun, mais par une affirmation spécifique qui irait à l'encontre de cet intérêt commun ${ }^{5}$. Par conséquent, plus il y a de grandes fortunes dans un État, plus les forces centrifuges s'expriment avec puissance dans le sens de la désaffection par rapport au bien général; or la taille des fortunes croît du même pas que la différenciation qui accompagne toujours la croissance de la taille de l'État.

4. Montesquieu, De l'esprit des lois, Genève, 1748, liv. VIII, chap. xvi (cité par la suite comme $E L$, les livres seront cités en chiffres romains et les chapitres en chiffres arabes).

5. Cf. Samuel H. BEER, To make a Nation. The rediscovery of American federalism, Harvard, Harvard University Press, 1993, p. 219 sq. 
Les propriétaires de ces grandes fortunes auront «peu de modération ", c'est-à-dire qu'ils seront peu disposés à modérer leurs propres intérêts ou à y renoncer en partie pour faire place à l'intérêt commun; les forces de la particularité sont donc beaucoup plus puissantes dans une société fortement différenciée que là où les conditions sont relativement égales et où, dans cette mesure, les citoyens sont disposés à renoncer à une partie de leur intérêt particulier pour se mettre en accord avec d'autres. Lorsque les fortunes sont très inégales, la partie de l'intérêt des plus riches qui est spécifique et non pas commune avec l'intérêt des plus pauvres - devient déterminante : c'est à ce moment-là que les propriétaires de grandes fortunes veulent une politique favorable à leurs intérêts particuliers (la section de leurs intérêts par laquelle ils sont distincts ou même ennemis de leurs concitoyens) et non à l'intérêt commun.

Pour Montesquieu, il ne peut donc y avoir de république que là où la section commune de nos intérêts l'emporte en importance sur la section « privée » par laquelle les riches ( $\mathrm{s}$ 'il y en a) se distinguent des pauvres. C'est assez dire que les républiques doivent être fondées sur la vertu, c'est-à-dire sur la disposition des individus à renoncer à ce qui fait d'eux des particuliers pour ne penser qu'à l'intérêt commun; elles ne sont donc possibles que là où les intérêts divergent peu, en sorte que ce à quoi les plus riches doivent renoncer pour adhérer à l'intérêt commun est peu important au regard de leur intérêt considéré dans son ensemble; mais la vertu devient bien évidemment impossible là où elle exigerait des plus riches qu'ils renoncent à la majeure partie de leur intérêt (toute la section « privée » ou particulière) pour adhérer au bien public et en faire le motif dominant de leurs actions.

Dès lors, les républiques sont nécessairement de petite taille, et elles peuvent être démocratiques à cause de la (relative) communauté d'intérêts entre leurs membres. Elles n'ont pas besoin de réfléchir sur les moyens institutionnels permettant d'obtenir que, en l'absence de motif vertueux c'est-à-dire en l'absence d'un sentiment d'identification de chacun au bien public - les individus puissent néanmoins être déterminés - artificiellement - à agir pour le bien de l'ensemble; elles n'ont donc pas besoin de se demander comment suppléer l'absence d'un motif vertueux défaillant, car celui-ci existe lorsque les conditions sont approximativement égales. Elles peuvent compter sur la vertu des plus nobles ou des plus riches (qui ne le sont jamais que de manière toute relative), et sur le dévouement des hommes d'exception à qui les récompenses honorifiques pourront servir de substitut à la petite part de leur intérêt qu'ils sacrifient en servant le bien général. De tels hommes d'élite sauront donc enseigner à leurs concitoyens, par la parole et par l'exemple, les voies de l'abnégation, au demeurant pas si héroïque, et fort bien compensée par les honneurs lorsque les fortunes sont un tant soit peu égales. 
Mais lorsque ces républiques atteignent une trop grande étendue, ce dévouement des hommes supérieurs leur fait défaut à mesure que les écarts de condition se creusent et que les hommes supérieurs sont tentés de jouer leur propre jeu, parce que la part de leur intérêt qu'ils devraient sacrifier s'est accrue dans des proportions que l'étendue des honneurs qu'ils peuvent attirer sur eux dans une grande cité ne saurait compenser efficacement. Elles périssent lorsqu'elles atteignent une trop grande étendue, et que les richesses s'y accumulent au point que l'inégalité des conditions matérielles y rend les riches sourds aux appels de l'intérêt général que dispensent les hommes vertueux.

Ainsi, pour Montesquieu, des hommes ne peuvent s'accorder que si leurs conditions sociales et juridiques sont relativement homogènes. Lorsqu'elles deviennent hétérogènes, il n'est plus possible d'avoir un accord et il est inévitable que les plus forts oppriment les plus faibles. Dans un pays qui connaît cette diversité hétérogène de conditions, la politique ne doit donc pas chercher l'harmonisation par la discussion, mais l'équilibre sous la forme d'un système de contrepoids entre des intérêts qui demeurent divergents. Dès lors, elle ne peut plus être intégralement démocratique, mais le poids spécifique du nombre (les pauvres désormais devenus un intérêt spécifique) doit être contrebalancé par un autre élément (les élites).

La politique au sein d'un grand État ne peut donc être que l'art par lequel chacun cherche à promouvoir ses propres intérêts par tous les moyens; elle a cessé d'être le lieu de la modération de l'intérêt privé au bénéfice de l'état public; il ne s'agit plus de renoncer à une partie de l'intérêt qui nous anime pour qu'il puisse coïncider avec celui des autres, mais de l'emporter sur celui des autres. Bref, la vertu y est devenue impossible, et c'est une simple conséquence de l'inégalité extrême; cette inégalité extrême est, à son tour, une conséquence de la taille de l'État.

Dans ce contexte, les forces et les intérêts vont chercher à se coaliser pour triompher de coalitions plus faibles ou pour opprimer des intérêts devenus minoritaires ${ }^{6}$. Dans un grand pays, la prépondérance de la libre affirmation des intérêts de chacun débouche donc facilement sur l'oppression de la minorité par la majorité; c'est la raison pour laquelle le régime républicain ne convient pas à un grand pays : il risquerait, à cause de l'importance qu'il accorde à l'expression de la volonté de chacun, de déboucher sur une forme de tyrannie de la majorité.

La république est donc funeste aux grands États, où le peuple ne doit pas paraître en personne sur la scène politique, mais où l'on doit au contraire inventer des procédures destinées à canaliser la libre expression des inté-

6. Cf. Thomas L. Pangle, Montesquieu's philosophy of liberalism, Chicago, Chicago University Press, 1973, p. 123-125. 
rêts, qui ne demandent qu'à se coaliser et à s'opprimer les uns les autres; c'est pourquoi la volonté populaire doit être freinée et privée pour ainsi dire de toute souveraineté sans partage.

Montesquieu affirme ainsi qu'il est impossible que le peuple ait luimême la puissance législative dans un grand État et que, même dans un petit État, cela est sujet à beaucoup d'inconvénients. Il faut donc que le peuple fasse ce qu'il a à faire par ses représentants ${ }^{7}$. De même, il est impossible que le peuple prenne des résolutions actives et qui demandent quelque exécution, car il en est incapable. Dans tout État, il y a des élites et des hommes de talent (« des gens distingués par la naissance, les richesses ou les honneurs ») et ils doivent disposer d'un pouvoir propre, faute de quoi ils seraient opprimés par la majorité :

« [...] mais s'ils étaient confondus parmi le peuple, et s'ils n'y avaient qu'une voix comme les autres, la liberté commune serait leur esclavage, et ils n'auraient aucun intérêt à la défendre, parce que la plupart des résolutions seraient contre eux. La part qu'ils ont à la législation doit donc être proportionnée aux autres avantages qu'ils ont dans l'État : ce qui arrivera s'ils forment un corps qui ait droit d'arrêter les entreprises du peuple, comme le peuple a droit d'arrêter les leurs ${ }^{8}$."

Montesquieu refuse donc tous les dispositifs destinés à promouvoir une démocratie plus ou moins directe : les mandats impératifs, la permanence des sessions de l'assemblée qui représente le peuple, ainsi que le droit qu'elle pourrait avoir de s'assembler de sa propre initiative. À l'époque moderne, la démocratie menace plus que jamais d'être le règne d'une faction - le plus grand nombre, les pauvres - et une simple tyrannie de la majorité; on ne peut y faire obstacle qu'en introduisant une restriction de la notion même de gouvernement par le grand nombre.

Puisque le peuple est incapable de se gouverner lui-même sans céder à l'esprit factionnel et partisan, il doit être confiné au choix de ses représentants :

« Le peuple est admirable pour choisir ceux à qui il doit confier quelque partie de son autorité. Il n'a à se déterminer que par des choses qu'il ne peut ignorer, et des faits qui tombent sous les sens [...] Mais saura-t-il conduire une affaire, connaître les lieux, les occasions, les moments, en profiter? Non, il ne le saura pas [...] le peuple, qui a assez de capacité pour se faire rendre compte de la gestion des autres, n'est pas propre à gérer par lui-même ${ }^{9}$."

Dans un grand État, les ambitions et les désirs de domination ont cessé d'être pernicieux : ce sont eux qui donnent vie à cette forme de gouverne-

7. $E L, \mathrm{XI}, 6$.

8. $E L, X I, 6$.

9. $E L, \mathrm{II}, 2$. 
ment, car les hommes ne sont poussés à agir que par la recherche de leur intérêt particulier, et non plus par le souci de la patrie (qui ne peut plus exister quand celle-ci est devenue trop vaste, et que l'étendue des jouissances privées fait que l'exercice des fonctions politiques et militaires a cessé d'être la voie principale de la satisfaction). L'ambition, ici, a cessé d'être dangereuse parce qu'elle se heurte sans cesse à une ambition contraire, comme si l'universalisation des passions les rendait capables de se neutraliser les unes les autres. À l'instar d'un système mécanique, l'ambition de chacun agit comme une force qui le fait diverger de l'intérêt général, alors que le contrepoids des ambitions des autres le contraint à revenir vers ce même intérêt; il y a une sorte de main invisible qui ramène chacun dans le droit chemin par souci de sa propre réputation; c'est ce que Montesquieu appelle l'honneur :

«Vous diriez qu'il en est comme du système de l'univers, où il y a une force qui éloigne sans cesse du centre tous les corps, et une force de pesanteur qui les y ramène. L'honneur fait mouvoir toutes les parties du corps politique; il les lie par son action même; et il se trouve que chacun va au bien commun, croyant aller à ses intérêts particuliers.

Il est vrai que, philosophiquement parlant, c'est un honneur faux qui conduit toutes les parties de l'État; mais cet honneur faux est aussi utile au public que le vrai le serait aux particuliers qui pourraient l'avoir ${ }^{10}$."

Cette main invisible s'applique au problème des élites : en faisant effort pour leurs propres intérêts, les hommes d'élite contrebalancent la toutepuissance de l'élément démocratique et aident le système à se maintenir dans l'état d'équilibre favorable à la liberté de tous. La corruption anglaise, c'est-à-dire le système par lequel l'exécutif empêche les communes de parvenir à une forme de toute-puissance législative grâce à l'emploi des placemen qui brident la puissance de l'assemblée, devient ainsi une nécessité politique et un rouage du bon gouvernement ${ }^{11}$.

On aboutit à un modèle mécanique explicite chez Montesquieu : la politique est l'art par lequel on balance des intérêts les uns par les autres. À l'inverse de la politique des Anciens, qui cherche à faire que la particularité des intérêts soit en quelque sorte transcendée, la politique moderne accepte que les intérêts demeurent ce qu'ils sont : égoïstes et désespérément particuliers; plutôt que de les appeler à se dépouiller de leur particularité, elle cherche à neutraliser celle-ci en les opposant ingénieusement les uns aux autres :

10. EL, III, 7.

11. C'est aussi l'opinion de David Hume; cf. "Of the independency of Parliament" et "Whether the British government inclines more to absolute monarchy or to a republic ", in Essays, moral, political and literary, Indianapolis, Liberty Fund, 1985, p. 42-53. 
" Pour former un gouvernement modéré, il faut combiner les puissances, les régler, les tempérer, les faire agir ; donner, pour ainsi dire, un lest à l'une pour la mettre en état de résister à une autre : c'est un chef-d'œuvre de législation que le hasard fait rarement, et que rarement on laisse faire à la prudence ${ }^{12}$. "

La liberté moderne passe par la connaissance de cet art du gouvernement, qui consiste à équilibrer les différentes forces intangibles qui composent un État de grande dimension. Chaque intérêt conserve sa propre direction et demeure motivé par ses propres valeurs, lesquelles donnent à ses membres un puissant intérêt commun contre les autres intérêts; chaque intérêt demeure également isolé des autres et sans communication avec eux, mais protégé aussi par la constitution qui empêche qu'il soit submergé par une coalition d'intérêts contraires.

Cette politique est « conservatrice "; ce n'est pas un outil de recherche de l'intérêt collectif et de promotion de ce même intérêt, mais un outil de préservation et de défense des intérêts tels qu'ils sont. Elle fait face à la divergence des intérêts par l'isolement et les contrepoids. Il n'est donc plus du tout question d'un « gouvernement par la discussion », où les citoyens tenteraient de dépasser ce qui les particularise et les oppose pour se placer du point de vue de l'intérêt général : les écarts entre les différents intérêts en présence ne le permettent tout simplement plus. Dès lors, la politique n'est plus une réflexion sur la légitimité mais un calcul sur la compatibilité; elle n'est plus fondée sur le refoulement du règne de la force au profit d'une règle acceptée par tous comme légitime, mais sur l'art d'équilibrer les forces les unes par les autres ${ }^{13}$.

C'est dans ce contexte qu'il faut situer le reproche que Montesquieu adresse à James Harrington ${ }^{14}$; ce reproche est dépourvu de tout fondement car l'auteur d'Oceana refusait de voir dans la constitution britannique un exemple du «plus haut point de liberté où la constitution d'un État peut être portée ». À ses yeux, la liberté d'une constitution se reconnaît non à sa capacité d'équilibrer judicieusement les forces les unes par les autres, mais à sa faculté de provoquer la confrontation maîtrisée des intérêts pour les amener à abandonner ce qui les particularise dans le cours d'une délibération portant sur l'intérêt commun.

Montesquieu fait donc se croiser deux thèmes, l'un très ancien, l'autre plus récent : le premier est l'incapacité du peuple à se gouverner lui-même;

12. $E L, \mathrm{~V}, 14$.

13. Cf. Maurizio VIROLI, From politics to reason of State. The acquisition and transformation of the language of politics, 1250-1600, Cambridge, Cambridge University Press, 1992.

14. EL, XI, 6 : « Harrington, dans son Oceana, a aussi examiné quel était le plus haut point de liberté où la constitution d'un État peut être portée. Mais on peut dire de lui qu'il n'a cherché cette liberté qu'après l'avoir méconnue, et qu'il a bâti Chalcédoine, ayant le rivage de Byzance devant les yeux. » 
le second est l'idée que cet inconvénient est plus grand encore dans les Temps modernes parce que la diversité des intérêts au sein de l'État provoque des coalitions et débouche sur la tyrannie de la majorité : le gouvernement populaire pur y est donc impossible et le pouvoir du peuple doit toujours y être contrebalancé, équilibré par un pouvoir qui en est plus ou moins indépendant.

\section{MACHIAVEL ET LE POUVOIR DU PEUPLE}

Machiavel avait pour ainsi dire répondu par avance à l'accusation de Montesquieu lorsque, dans les Discours sur la première décade de TiteLive, il s'était employé à réfuter l'idée que le peuple est incapable de se gouverner lui-même. Pour lui, cette idée est tellement répandue qu'elle en est presque évidente : le peuple est mobile, léger, inconstant, en sorte qu'il est voué à servir avec bassesse ou à dominer avec insolence ${ }^{15}$. Mais il ajoute que cette impression se dissipe lorsqu'on considère que les princes, lorsqu'ils ne sont pas retenus par le frein des lois, ne sont pas moins sujets à s'égarer que les peuples dans la même situation :

" Je conclus donc contre l'opinion commune qui veut que le peuple, lorsqu'il domine, soit léger, inconstant, mobile, ingrat; et je soutiens que ces défauts ne sont pas plus naturels aux peuples qu'aux princes. Les en accuser également est vérité; en excepter les princes, c'est erreur, car un peuple qui commande et qui est réglé par des lois est prudent, constant, reconnaissant autant et même à mon avis plus qu'un prince même réputé sage. D'un autre côté, un prince dégagé du frein des lois sera ingrat, changeant, impudent même, plus qu'un peuple placé dans les mêmes circonstances que lui. La différence de nuance qui existe entre eux ne vient pas de la diversité de leur naturel, qui est absolument le même, et qui ne pourrait avoir que des différences à l'avantage du peuple, mais bien du plus ou moins de respect que le peuple et le prince ont pour les lois sous lesquelles ils vivent. Or, si vous examinez le peuple romain, vous le verrez pendant quatre cents ans ennemi de la royauté, passionné pour le bien public et pour la gloire de la patrie : mille exemples appuient cette vérité ${ }^{16}$."

Machiavel montre ensuite que le peuple se montre invariablement plus sage dans le choix des hommes que les princes, à qui il est facile de persuader d'élever aux plus grands honneurs des individus sans la moindre

15. Machiavel, Discours sur la première décade de Tite-Live, 1532, Paris, Flammarion (« Champs »), 1985, liv. I, chap LvIII.

16. Ibid. 
valeur; le peuple est également plus constant dans ses décisions lorsqu'il est mis hors d'état d'en changer sans passer par des formes prescrites et rigoureuses. Au demeurant, les peuples font aussi des erreurs, mais celles-ci sont plus aisément remédiables que celles des princes, car quelques mots sages et bien placés suffisent souvent pour ramener à la raison une multitude qui s'égare, au lieu que le fer et la violence sont souvent les seuls remèdes contre les emportements tyranniques des princes.

«Un homme de bien peut souvent par son éloquence ramener un peuple licencieux et mutin; mais nul ne peut faire revenir un prince et l'on n'a d'autre moyen que la force. Que l'on juge de la gravité de leurs maladies respectives par la différence des remèdes. Pour guérir celle du peuple, il y faut souvent quelques paroles; pour guérir celle du prince, il faut toujours employer le fer; lequel de ces deux maux jugera-t-on le plus dangereux ${ }^{17}$ ? ”

Enfin, le mal qu'un peuple peut faire existe surtout par les conséquences de ses actions désordonnées qui peuvent aboutir à la tyrannie, au lieu que le mal d'un prince est présent dans ses actions elles-mêmes ${ }^{18}$. Ce n'est donc pas la démocratie qui, par elle-même, est source d'égarements, d'esprit factionnel et de triomphe de l'intérêt partisan du plus grand nombre sur l'intérêt général, mais le fait que la multitude puisse exprimer ses passions et faire prévaloir sa volonté sans rencontrer le frein des lois. Dès lors, le remède ne consiste pas à équilibrer le pouvoir du peuple par celui des élites, mais à contraindre ce pouvoir à ne s'exercer que de manière légale et selon les formes prescrites.

Reprenant l'exemple de Machiavel, Harrington montre lui aussi qu'il ne s'agit pas de limiter la volonté populaire, ni de pondérer l'élément de démocratie dans le gouvernement, et il n'adhère nullement à l'idée que le gouvernement par le grand nombre mène nécessairement à la tyrannie de la majorité, ou que les passions populaires vont nécessairement y prévaloir. Tout au contraire, si l'on est capable de fixer des règles par lesquelles doit s'exprimer la volonté de la majorité, le gouvernement par le grand nombre peut devenir le meilleur instrument de promotion de l'intérêt public, et non

\section{Ibid.}

18. Rousseau notera de la même manière qu'il y a cette différence entre les erreurs du peuple et celles des princes que les premières tournent toujours au prejudice de ceux qui les commettent, au lieu que les secondes tournent non pas au préjudice du prince qui s'en rend responsable mais à celui de son peuple; voir Rousseau, Lettres écrites de la Montagne, 1764, lettre IX, in Euvres complètes, t. III, Paris, Gallimard (« Bibliothèque de la Pléiade »), 1964, p. 891 : «Supposons l'abus de la liberté aussi naturel que celui de la puissance. Il y aura toujours cette différence entre l'un et l'autre que l'abus de la liberté tourne au préjudice du peuple qui en abuse et, le punissant de son propre tort, le force à en chercher le remède; ainsi, de ce côté le mal n'est jamais qu'une crise, et il ne peut faire un état permanent. Au lieu que l'abus de la puissance ne tournant point au préjudice du puissant mais du faible, est par sa nature sans frein, sans mesure, sans limite; il ne finit que par la destruction de celui qui le ressent. " 
plus celui de la prévalence d'un intérêt sur les autres. Il suffit pour cela d'inventer des règles pour l'expression de la volonté populaire. Comme dans l'exemple des deux petites filles qui ont à se partager un gâteau, on pourra parvenir à la justice par une procédure appropriée : si le sénat délibère tandis que l'assemblée décide, on aura la garantie que les lois ne seront pas oppressives ${ }^{19}$.

\section{LA RÉPONSE DE JAMES MADISON}

Dans Le Fédéraliste, Madison reprend cette idée, afin d'établir que le pouvoir du peuple n'a pas à être «équilibré » par un autre qui n'en serait pas issu. On peut construire une république d'une manière telle que tous les pouvoirs viennent du peuple et lui appartiennent, sans que cela provoque le règne tant redouté du factionnalisme et de l'esprit partisan, ni la tyrannie de la majorité qui paraît à beaucoup la conséquence inéluctable de la toutepuissance politique de la «multitude». Le peuple d'un grand État, dit Madison, est très capable de se gouverner lui-même à condition de le faire non pas directement mais par l'intermédiaire de ses représentants ${ }^{20}$.

Madison récuse donc la thèse - défendue par les antifédéralistes ${ }^{21}$ selon laquelle la constitution républicaine ne pourrait exister que dans des États de petite taille, tandis que, dans les grands États, la diversité des intérêts et l'inégalité des fortunes transformeraient nécessairement le gouvernement populaire, sous la menace des factions et de la tyrannie de la majorité, en un règne des élites qui entraverait et limiterait sévèrement le pouvoir du peuple. L'hypothèse de l'«aristocratisation » nécessaire des grandes républiques est un fantasme dépourvu de tout fondement, car ce n'est au contraire que dans ce genre d'États que la démocratie peut être guérie de ses propres excès, alors même que, dans les petits États, elle finit toujours par succomber à ses démons et par sombrer dans l'anarchie et la tyrannie.

Madison renverse donc le préjugé commun, qui est aussi celui de Montesquieu : la république, dit-il, ne convient qu'à de vastes territoires car ce n'est que là qu'elle peut neutraliser l'effet des passions populaires qui emportent tout sur leur passage dans les petites cités, et qui finissent par y

19. James Harrington, Océana, Préliminaires, éd. John Greville Agard Pocock, trad. franç., Paris, Belin, 1995, p. 244.

20. Alexander Hamilton, John JAY, James Madison, Le Fédéraliste, trad. franç., Paris, Librairie générale de droit et de jurisprudence, 1957.

21. Cf. Herbert Storing, «What the antifederalists were for?", in The Complete AntiFederalist, 7 vol., Chicago, Chicago University Press, 1981, vol. 1. 
tuer le gouvernement populaire. Cette thèse suppose la distinction entre une république - régime où tout le pouvoir vient du peuple - et une démocratie - régime où tout le pouvoir est exercé par le peuple ${ }^{22}$. Lorsque le peuple se dote d'un gouvernement républicain et représentatif, il est faux qu'il soit incapable de se gouverner lui-même et qu'il soit nécessairement factieux et inconstant, tyrannique et inconsidéré.

Cette distinction permet à son tour de rejeter l'idée de la constitution mixte, selon laquelle le pouvoir du peuple devrait être équilibré par celui des meilleurs; cette thèse fondamentale des « théories élitistes » n'a rien à voir avec le concept de république, qui n'est certainement pas un effort pour organiser la séparation des pouvoirs de manière à ce que la puissance du peuple soit contrebalancée par un pouvoir qui n'émane pas de lui. Tout au contraire, la république est une tentative pour permettre que tous les pouvoirs émanent du peuple et de lui seul, sans qu'il en résulte les inconvénients afférents à la démocratie directe. Le secret consiste à balancer ces pouvoirs les uns par les autres pour empêcher qu'aucun d'entre eux ne puisse devenir la proie de la simple volonté majoritaire et de l'esprit de faction.

Contre l'idée - chère aux antifédéralistes - que la seule manière de pallier la puissance insuffisante des petites républiques est de créer des confédérations d'États indépendants (faute de quoi on tombe dans l'inconvénient des grands États) qui ne s'associent que pour leur défense extérieure, Madison fait donc valoir que l'union en un seul grand corps permettra de faire coup double : pallier la faiblesse extérieure, tout en prémunissant le gouvernement populaire contre les maux qui le guettent lorsqu'il demeure confiné dans de petits États.

Pourquoi, selon Madison, les risques de tyrannie de la majorité sont-ils plus importants dans une petite république que dans une grande? Le diagnostic est simple : parce que la volonté populaire y prévaut de manière trop immédiate, sans freins ni filtres, et sans s'embarrasser de règles de manifestation et de contrôle. Ainsi les petites républiques que sont les treize États composant la confédération sont affectées de nombreux maux : instabilité, injustices, confusion, oubli du bien public au profit des intérêts factionnels, domination de l'avantage immédiat, etc. De plus, dit Madison,

« Les questions sont trop souvent décidées non pas d'après les règles de la justice et les droits de la minorité, mais par la force supérieure d'une majorité intéressée et oppressive ${ }^{23}$.»

22. Cf. Gordon S. Wood, La Création de la république américaine: 1776-1787, trad. franç., Paris, Belin, 1991.

23. J. Madison, Le Fédéraliste, op. cit. supra n. 20, n ${ }^{0} 10$, p. 67. 
Une telle situation fait qu'on ne peut guère se fier aux engagements publics et que les droits privés sont constamment menacés. "Ce sont là, ajoute Madison, les effets de l'instabilité et de l'injustice dont un esprit de faction a souillé nos administrations publiques. »

Une faction, c'est un ensemble de citoyens unis par un intérêt commun qui est à la fois contraire aux intérêts des autres citoyens et aux intérêts permanents et généraux de la communauté dans son ensemble; c'est donc un ensemble de citoyens rassemblés autour d'un intérêt qui est clairement particulier. Cet esprit factionnel est-il consubstantiel au gouvernement populaire (comme le soutiennent les ennemis de cette forme de gouvernement)? Et si ce n'est pas le cas, comment séparer le bon grain de l'ivraie?

Les remèdes imaginables ne sont pas légion. On pourrait tout d'abord tenter de s'attaquer aux causes mêmes des factions, et il y aurait pour cela deux moyens ${ }^{24}$. Le premier serait pire que le mal puisqu'il consisterait tout simplement à supprimer la liberté dont les factions se nourrissent. Le second serait de mettre tous les citoyens dans des conditions si égales et si homogènes que leurs intérêts seraient en quelque sorte identiques; on couperait ainsi l'herbe sous le pied des rivalités factionnelles. C'est ce « moyen » que, aux yeux de Montesquieu, les cités antiques pratiquaient par le fait, et chacun comprend aisément qu'il n'est accessible qu'aux États de petite dimension. Il permet certes de penser que le gouvernement républicain n'est pas essentiellement générateur d'esprit factionnel, mais il nous enjoint cependant de conclure que cette forme de gouvernement ne peut pas convenir à de grands États, où les conditions sont de plus en plus hétérogènes.

Faut-il en rester là et voir dans cette homogénéité des conditions la qualité impérative de la survie des régimes républicains? Si c'était le cas, il faudrait effectivement en rester à la confédération des treize républiques. Madison soutient qu'il n'en est rien car, selon lui, une telle convergence des intérêts par l'homogénéisation des conditions est impraticable même dans les États de petites dimensions :

« Tant que la raison de l'homme sera faillible, et que l'homme aura la faculté de l'exercer, il se formera des opinions divergentes; tant qu'il y aura des rapports entre sa raison et son amour-propre, ses opinions et ses passions auront les unes sur les autres une influence réciproque, et celles-ci seront les objets auxquels celles-là s'attacheront ${ }^{25}$. $\gg$

24. Cf. Jennifer Nedelsky, Private Property and the limits of American constitutionalism. The Madisonian framework and its legacy, Chicago, Chicago University Press, 1990, p. 165166.

25. J. Madison, op. cit. supra n. 23, p. 68. 
La diversité prend sa source dans la nature humaine au point que, pour faire durablement converger les intérêts de tous, il faudrait changer les hommes en des créatures d'une autre espèce. La société civile elle-même trouve sa raison d'être dans ces différences, et sa fonction n'est pas de les supprimer, mais au contraire de maintenir cette diversité bénéfique et d'en protéger l'épanouissement :

« De la protection des facultés différentes et inégales pour l'acquisition de la propriété résulte immédiatement l'inégalité dans l'étendue et la nature des propriétés; et de leur influence sur les sentiments et les opinions des propriétaires respectifs, résulte la division de la société en intérêts différents et en partis différents ${ }^{26}$.

La société n'existe donc que pour la protection des différences entre les individus, avec tous leurs effets, parmi lesquels figurent aussi la diversité des facultés, des propriétés et des conditions, ainsi que l'opposition d'intérêts qui en est la suite inéluctable ${ }^{27}$.

Pour Madison, une telle « variété » est souhaitable, non seulement dans les conditions matérielles (parce qu'elle est gage de prospérité), mais aussi dans les croyances philosophiques et religieuses (où elle est gage de progrès vers la vérité). L'homogénéité nous condamnerait au contraire à la stagnation sur ces deux plans ${ }^{28}$.

Dès lors, le remède aux maux du gouvernement populaire (l'homogénéité des conditions censée supprimer les factions) serait en contradiction avec la finalité même de tout gouvernement, qui est la coexistence paisible entre les intérêts différents, et non la suppression de ces mêmes différences au profit d'une illusoire homogénéité ${ }^{29}$. La variété étant pour ainsi dire coextensive à l'existence humaine, il est vain de vouloir s'en affranchir ou la supprimer :

« Si puissant est le penchant qui entraîne les hommes dans des animosités mutuelles que, lorsqu'il ne se présente pas d'occasions importantes, les distinc-

26. Ibid., p. 68-69.

27. Cf. Bernard BAILYN, Faces of Revolution, personalities and themes in the struggle for American independance, New York, Belknap Press, 1990, p. 258-259.

28. L'influence de Milton est ici clairement décelable; cf. John Mllton, Areopagitica. A speech for the liberty of unlicensed printing to the parliament of England, Londres, 1644.

29. J. MAdison, op. cit. supra n. 23, p. 69 : «L'intérêt de l'agriculture, l'intérêt des manufactures, l'intérêt du commerce, l'intérêt des capitalistes, et d'autres intérêts moins importants, se forment nécessairement dans les nations civilisées et les divisent en différentes classes qui agissent d'après des vues et des sentiments différents. Le règlement de cette multitude d'intérêts opposés, voilà le principal but de la législation moderne; l'esprit de parti et de faction entre toujours aujourd'hui dans le calcul des opérations ordinaires et nécessaires du gouvernement. » 
tions les plus frivoles et les plus fantastiques ont suffi à réveiller leurs passions ennemies et à exciter entre eux les plus violents conflits. Mais la source de factions la plus commune et la plus durable a toujours été l'inégale distribution de la richesse. Ceux qui possèdent et ceux qui ne possèdent pas ont toujours eu des intérêts différents. Les créanciers et les débiteurs ont entre eux une semblable ligne de démarcation ${ }^{30}$."

Vouloir traiter les causes des factions, c'est donc vouloir supprimer la civilisation, ramener les hommes à cet âge barbare où il n'y a aucune diversité d'intérêts parce que les hommes n'ont pas encore exercé leurs facultés ni employé celles-ci à la mise en ouvre des ressources de la nature.

Faut-il en conclure que tout gouvernement populaire est nécessairement vicieux et que la politique doit toujours avoir la forme de la répression? Le seul remède serait-il de tempérer la démocratie par l'aristocratie, et d'équilibrer les turbulences des factions populaires par la constitutionnalisation de l'intérêt séparé des élites? C'était la thèse des partisans de la constitution mixte, et c'est aussi celle de Montesquieu.

Madison raisonne tout autrement : l'existence des factions n'est pas une raison de désespérer du gouvernement du peuple par le peuple, et ce n'est pas une raison pour ôter à ce dernier le pouvoir suprême dans l'État, ni pour l'équilibrer par la représentation séparée des élites, puisqu'il est possible de maîtriser les effets des factions par une organisation adéquate des institutions.

Le secret de la réussite réside précisément dans la taille de l'État ${ }^{31}$. Renversant la théorie traditionnelle, qui affirmait que seuls des États de petite taille pourraient échapper aux maux engendrés par les factions populaires parce qu'ils jouiraient d'une population suffisamment homogène, Madison va montrer que ce n'est que dans des États de grande dimension que les effets des factions peuvent être maîtrisés, et que le gouvernement populaire ne débouche pas sur la tyrannie de la majorité. Autrement dit, loin qu'un grand État ne puisse pas être gouverné de manière républicaine et populaire, Madison va affirmer que seuls les grands États le peuvent, alors que les petits en sont incapables (outre que ces derniers sont par définition incapables de se défendre contre les agressions extérieures).

Comment Madison raisonne-t-il? Dans une petite république, dit-il, l'homogénéité des conditions n'est jamais parfaite en sorte qu'il y a toujours au moins deux intérêts dont le plus nombreux ou le plus puissant l'emporte sur l'autre. Plus les dimensions de l'État décroissent, plus le nombre des intérêts se restreint sans jamais que la différence disparaisse complètement; dès lors, la diminution de la diversité des intérêts qui résulte

30. Ibid.

31. J. NEDELSKY, op. cit. supra n. 24, p. 158. 
de la diminution de la taille représente un danger pour la liberté, car là où il n'y a que deux intérêts, l'un possède nécessairement la majorité pour lui ${ }^{32}$. En revanche, dans une grande république où les intérêts différents sont plus nombreux, il est difficile à l'un d'entre eux de représenter une majorité et de devenir tyrannique ${ }^{33}$. Comme le dit Madison, rien n'est perdu lorsqu'une faction ne peut pas avoir à elle seule la majorité :

«Quand une faction ne forme pas une majorité, le remède existe dans le principe du gouvernement républicain, qui permet à la majorité de renverser ses sinistres projets par un vote régulier. La faction peut embarrasser l'administration, elle peut ébranler la société ; mais elle est incapable d'exécuter ses violences et de les cacher sous les formes de la Constitution ${ }^{34}$. "

Mais il n'en va évidemment pas de même lorsque la faction est majoritaire, ce qui peut se produire dans un petit État; elle s'empare alors du gouvernement de forme républicaine pour faire prévaloir ses projets particuliers, et elle se livre à l'oppression sous couleur de légalité :

«Quand une faction comprend la majorité, la forme du gouvernement populaire peut lui fournir les moyens de sacrifier à sa passion ou à ses intérêts dominants le bien public ainsi que les droits des autres citoyens ${ }^{35}$. »

La possibilité de défendre les droits individuels dans un gouvernement de forme républicaine existe donc si aucune des factions en présence n'est en mesure de s'emparer de la majorité; or ceci n'est possible que dans un

32. J. Madison, Le Fédéraliste, op. cit. supra n. 20, $\mathrm{n}^{\circ} 51$, p. $431:$ « Dans une société où la faction la plus puissante peut aisément se réunir et opprimer la plus faible, on peut dire que l'anarchie règne aussi bien que dans l'état de nature, où l'individu le plus faible n'est pas à l'abri de la violence du plus fort. "C'est le cas dans une démocratie de petites dimensions, où l'un des intérêts en présence peut aisément être majoritaire et s'emparer des institutions de l'État pour opprimer l'intérêt le moins fort. Madison montre que cette oppression légale d'un intérêt par un autre est une sorte d'état de nature et que, comme ce dernier, elle se résout nécessairement par l'appel à un tyran capable de protéger toutes les parties en présence, voir ibid. : "Et de même que, dans l'état de nature, les individus plus forts sont eux-mêmes contraints par l'incertitude de leur condition à se soumettre à un gouvernement qui protège les faibles ainsi qu'eux-mêmes, de même, dans un gouvernement anarchique, les mêmes motifs conduiront peu à peu les factions ou les partis les plus puissants à souhaiter un gouvernement qui protège tous les partis, les faibles et les forts. "

33. ID., ibid., $\mathrm{n}^{\circ} 10$, p. 75 : « Dans une république, il y a un nombre plus grand de citoyens et un territoire plus vaste que dans un gouvernement démocratique; et c'est particulièrement cette circonstance qui rend les plans des factieux moins redoutables dans la république que dans la démocratie. Moins une société est étendue et moins nombreux sont les partis et les intérêts différents qu'on y rencontre; moins il y a de partis et d'intérêts différents, et plus il y a de chances pour que le même parti ait la majorité, et plus est petit le nombre des individus qui composent la majorité, plus petite est l'enceinte qui la renferme, plus aisément elle peut concerter et exécuter ses plans d'oppression. »

34. Ibid., p. 71.

35. Ibid. 
vaste État, lorsqu'il y a une telle diversité de factions ou d'intérêts particuliers qu'aucun d'entre eux ne peut mettre à profit la forme populaire du gouvernement pour faire passer ses vues particulières par le canal de la loi; tout intérêt devra donc composer avec d'autres dans une coalition; or une coalition d'intérêts a moins de facilité pour mener à bien des menées tyranniques contre les minorités que les membres bien soudés d'un intérêt unique :

«Étendez sa sphère, elle comprendra une plus grande variété de partis et d'intérêts et vous aurez moins à craindre de voir à une majorité un motif commun pour violer les droits des autres citoyens; ou, s'il existe un tel motif commun, il sera plus difficile à ceux qui l'éprouvent de connaître leur propre force et d'agir de concert. Sans compter les autres obstacles, il est facile de voir que partout où se trouve la conscience d'un projet injuste et malhonnête, l'accord est toujours arrêté par la défiance, en proportion du nombre d'hommes dont le concours est nécessaire ${ }^{36}$."

La grande taille de l'État favorise ce genre d'effets de confrontation; la diversité des intérêts oblige chacun à tenir compte de ceux des autres puisqu'il est impossible à aucun d'entre eux de gouverner seul. Comme le dit Madison à propos de l'avantage qu'il y aurait à former une grande république :

"Cet avantage consiste dans la sécurité plus sérieuse qu'une plus grande variété de partis apporte contre la possibilité de voir un parti opprimer le reste de la société par la supériorité du nombre ${ }^{37}$."

Les petites républiques sont donc bien une contradiction dans les termes, et l'on doit en conclure que le gouvernement de forme républicaine est incompatible avec la petitesse de l'État :

« De tout ceci, il faut conclure qu'une démocratie pure, je veux dire une société composée d'un petit nombre de citoyens qui s'assemblent et se gouvernent eux-mêmes, ne comporte aucun remède contre les méfaits des factions. La majorité aura, presque dans tous les cas, des passions et des intérêts communs, la communication et l'accord résulteront nécessairement de la forme même du gouvernement; il n'y a rien qui puisse réprimer le désir de sacrifier le parti le plus faible ou un individu sans défense. Ainsi les démocraties pures de ce genre ont-elles toujours offert le spectacle du trouble et des dissensions; elles ont toujours été incompatibles avec la sûreté personnelle et le maintien des droits de propriété; elles ont eu en général une existence éphémère et une mort violente ${ }^{38}$."

36. Ibid.

37. Ibid., p. 76

38. Ibid., p. 72 . 
Si l'impossibilité de l'égalité absolue interdit toute élimination des factions par l'homogénéisation des membres de la société civile, la seule issue est donc de multiplier les factions pour empêcher qu'aucune d'entre elles ne puisse posséder la majorité; or cette tactique n'est possible que dans un État dont la population et les intérêts divers sont suffisamment nombreux.

Dans une grande république, le gouvernement populaire passe en outre de la démocratie directe à la démocratie représentative; cette représentativité, loin d'être une confiscation du pouvoir du peuple ou une manière de l'entraver, a seulement pour objet d'en raffiner l'expression et de lui permettre de survivre en se débarrassant de ses aspects les plus grossièrement factionnels. C'est par une illusion que les antifédéralistes ont pu croire que cette représentativité avait pour but de priver le peuple de son pouvoir effectif de décision dans les affaires communes; c'est tout le contraire qui est vrai, puisqu'elle a pour effet de rendre le pouvoir populaire moins factionnel et, par conséquent, d'en autoriser la conservation. Sans le caractère représentatif de la république, celle-ci verrait le peuple se livrer à ses appétits factionnels et aboutir tôt ou tard à la tyrannie.

Dans cette conception, le pouvoir du peuple n'est arrêté par rien : il n'a pas de contrepoids ; en particulier, il n'est pas équilibré, comme c'est le cas dans une constitution mixte, par un élément monarchique et par un élément aristocratique indépendants. Tous les pouvoirs, ceux du président, de la chambre basse et de la chambre haute, émanent du peuple et le représentent. Simplement, le pouvoir populaire est mis hors d'état de pouvoir céder à son factionnalisme «naturel »; il est obligé de passer par des canaux tels que la tyrannie de la majorité sur la minorité devient impossible, comme aussi l'oppression des droits des individus et les attaques contre la propriété.

Ici, la séparation des pouvoirs n'a plus du tout le sens qu'elle avait chez Montesquieu, puisqu'elle n'est plus qu'un moyen de rendre le gouvernement populaire à la fois possible dans la durée et plus efficace : elle est un moyen pour obliger le peuple, qui est l'unique intérêt présent sur la scène politique, à raffiner ses appétits, à discipliner ses factions, à fondre les différents particularismes qui coexistent en lui et à les confronter pour dégager l'intérêt commun.

Le républicanisme de Madison est donc un raisonnement pour répondre à toutes les théories élitistes de la démocratie; il permet de dire pourquoi l'intervention active du grand nombre ne débouche pas nécessairement sur la tyrannie de la majorité et sur l'inquisition. Lorsqu'on distingue la capacité constituante du peuple (qui signifie que tous les pouvoirs émanent de lui) de sa capacité gouvernante (qui signifie que le peuple gouverne par luimême), et lorsqu'une constitution adéquate contraint le peuple à ne gouver- 
ner qu'en confrontant et en raffinant les différents particularismes qui l'habitent, la démocratie cesse d'être la tyrannie de la multitude instable pour devenir un gouvernement stable et pondéré. Vouloir l'affranchir des filtres que sont la représentativité et la séparation des pouvoirs, c'est le condamner à sa perte.

Le républicanisme est aussi un raisonnement pour montrer que la politique n'est pas un système de rapports de forces en équilibre les unes avec les autres, mais un système institutionnel de confrontation et d'élimination réciproque des particularismes, un système d'élimination des factions en tant que telles, et une technique pour empêcher qu'elles ne s'emparent du gouvernement de l'État. C'est un moyen pour rechercher l'intérêt général, conçu non pas comme l'art de faire coexister différents intérêts sur le modèle mécanique de l'équilibre des forces, mais comme l'art de les faire discuter pour parvenir à un accord; c'est pour cette raison que le peuple a plusieurs séries de représentants. On ne cherche pas à obtenir que les différents intérêts, tout en demeurant ce qu'ils sont (c'est-à-dire des factions particulières), parviennent à un point d'équilibre et de dissuasion réciproque; on cherche, au contraire, à faire en sorte qu'ils se modifient dans le cours de la discussion à laquelle ils sont tenus de procéder, puisque aucun d'eux ne peut contrôler seul l'ensemble du pouvoir ${ }^{39}$. Ce gouvernement par la discussion est donc destiné à bannir le particularisme et à instaurer une règle qui soit vraiment à l'avantage de tous; il est destiné à bannir la forme de gouvernement - y compris la démocratie au sens premier du terme qui consiste simplement à consacrer par la loi le règne des plus forts.

Le républicanisme est donc opposé à l'idée que la politique est un art fondé sur la négociation; il ne s'agit pas, pour les différents intérêts particuliers en présence, d'abandonner une part de leurs exigences pour être en mesure de satisfaire les autres, dans un jeu gouverné par les rapports de forces où les plus puissants (ou les plus nombreux, ou les plus habiles) pourront imposer à leurs adversaires le marché qui leur apportera à euxmêmes les plus grands avantages. Il ne s'agit pas d'accepter de subir des lois que l'on sait voulues par des intérêts particuliers antagonistes (et donc que l'on estime mauvaises pour soi), afin d'être en mesure d'intégrer aux lois de l'État des règles favorables à nos propres intérêts particuliers; l'État ne peut pas être la chose de tous s'il est ainsi un tissu juxtaposé de règles qui favorisent tantôt un intérêt particulier et tantôt un autre, au gré des alliances électorales et de l'évolution des rapports de forces.

Dans l'optique de Madison, la république est justement une manière de tenir cette forme de négociation en lisière, pour la remplacer par la néces-

39. Cf. Bruce ACKerman, We the people. 1 : Foundations, Harvard, Harvard University Press, 1991. 
saire confrontation entre les intérêts particuliers et pour contraindre ces mêmes intérêts particuliers à s'entendre et à n'intégrer aux lois de l'État que les mesures qui sont favorables à l'intérêt général. En ce sens, le républicanisme est un antisectionnalisme; ce qui doit être présent dans l'État, ce ne sont pas des intérêts constitués, ni des communautés qui négocient savamment et habilement la promotion de leurs intérêts particuliers, mais des individus (et plus l'éclatement est grand mieux cela vaut) qui recherchent en commun les mesures qui seraient susceptibles d'être également favorables à tous. Si les individus s'engagent dans cette recherche, ce n'est pas par grandeur d'âme, mais parce que aucun d'entre eux ne peut accéder au pouvoir sans s'être réuni au préalable avec d'autres pour former une majorité, et parce que cette réunion entre tant d'intérêts divers ne peut avoir la forme d'un compromis négocié : elle passe nécessairement par le dépassement, au moins partiel, des intérêts spécifiques de chaque partenaire.

La solution ne consiste donc pas, pour Madison, à accorder aux élites un poids spécifique dans l'État et à leur permettre de jouer un rôle indépendant qui ferait contrepoids au peuple; cette solution n'en est pas une, car elle détruit le pouvoir populaire pour le sauver. La vraie solution consiste à se doter d'institutions républicaines dans un grand pays, et à faire que la multiplicité des intérêts et des factions les oblige à composer entre elles pour gouverner ${ }^{40}$.

La première méthode - celle qui consiste à créer une autorité indépendante de celle du peuple - existe dans tous les États qui ont une autorité héréditaire ou qui s'est nommée elle-même; Madison affirme que cette autorité indépendante, censée faire contrepoids à celle du peuple, n'est qu'une garantie précaire car, lorsque la majorité tentera d'opprimer la minorité, rien n'assure que cette autorité se rangera du côté de cette dernière et qu'elle s'emploiera à empêcher l'oppression : il se peut fort bien qu'elle favorise les projets injustes de la majorité, ou qu'elle ait des intérêts propres qui l'incitent à opprimer elle-même les deux partis.

La constitution des États-Unis offre un exemple de la seconde méthode, laquelle est proprement républicaine, car elle est l'art de concilier le gouvernement du peuple souverain et les garanties contre l'esprit de faction, la démocratie et les libertés individuelles :

40. J. MADISON, op. cit. supra n. 32, p. 432 : «Il existe nécessairement des intérêts divers dans les différentes classes de citoyens. Si la majorité est unie par un intérêt commun, les droits de la minorité seront en péril. Il n'y a que deux manières pour parer à ce danger; la première, c'est de créer dans la nation une volonté indépendante de la majorité, c'est-à-dire de la nation elle-même; la seconde, c'est de faire entrer dans la nation assez de classes différentes de citoyens pour rendre très improbable, sinon impossible, une combinaison injuste de la majorité. " 
« En même temps que toute autorité dans ce gouvernement dépendra de la nation, la nation elle-même sera divisée en un si grand nombre de partis, d'intérêts et de classes de citoyens, que les droits des individus ou de la minorité seront peu menacés par les combinaisons intéressées de la majorité. Dans un gouvernement, les droits civils doivent être défendus de la même manière que les droits religieux. Le moyen, c'est la multiplicité des intérêts dans un cas, et dans l'autre la multiplicité des sectes. Le degré de protection dans les deux cas dépendra du nombre des intérêts et des sectes; et l'on peut présumer que cela dépend de l'êtendue du pays et du nombre des habitants compris sous le même gouvernement ${ }^{41}$."

Madison montre donc que certains ont cru que, pour empêcher les factions de faire leurs ravages et d'aller donner dans la tyrannie, il fallait créer une volonté indépendante de la majorité et qui pourrait s'opposer à elle lorsque cela serait nécessaire; il rejette explicitement cette solution, car elle aboutirait à priver le gouvernement populaire de sa substance, et à réinstaurer le règne au moins partiel (puisqu'elles auraient le pouvoir d'arrêter la volonté populaire) des élites «naturelles ».

Certes, il y a et il y aura toujours des hommes d'élite; mais il est exclu qu'ils puissent constituer un intérêt séparé, distinct de celui du peuple. S'il en était autrement, on aurait une élite héréditaire, nécessairement fondée sur la naissance ou sur la richesse, qui constituerait un contrepoids au pouvoir du peuple; dans cette hypothèse, le principe de la souveraineté du peuple serait battu en brèche. Les seuls hommes d'élite que le gouvernement par discussion reconnaisse, ce sont les représentants du peuple, c'està-dire les hommes que le peuple a élevés à de hautes fonctions parce qu'ils ont rendu des services signalés à la cause commune et à l'intérêt général. Ce genre de services est et doit être, dans une république bien constituée, la seule manière de s'élever et de satisfaire la passion ambitieuse qui anime toujours les hommes particulièrement énergiques; ces passions n'ont rien d'illégitime en soi, mais on peut et on doit les canaliser pour qu'elles ne puissent recevoir satisfaction que de manière publique et pour des services rendus au public.

Au demeurant, comme le reconnaissait Machiavel, une république a besoin d'hommes qui sachent se distinguer; elle a besoin de leurs talents exceptionnels, en particulier pour être en mesure de percevoir suffisamment tôt les dangers qui menacent de la ruiner, ce que seuls peuvent faire des hommes exceptionnels; toute la difficulté consiste à obtenir de ces hommes d'exception qu'ils mettent leurs talents au service de la chose de tous et non pas de leurs intérêts particuliers. On peut l'obtenir en créant un

41. Ibid., p. 433. 
système institutionnel public et transparent dans lequel les hommes ne pourront s'élever que s'ils ont prouvé qu'ils consacraient leurs talents au service du bien commun; dans cette mesure, les deux parties seront également satisfaites sans pour autant que l'élite jouisse d'une représentation séparée ${ }^{42}$.

La première conclusion à tirer de cette distinction entre république et démocratie est que, comme l'avaient souligné John Milton et James Harrington, la confrontation des intérêts et leur discussion ne sont pas stériles : le débat a une vertu productrice de vérité et de progrès, en sorte que, sous l'influence de leur confrontation avec les intérêts d'autrui, les options et les intérêts des individus - ainsi que la manière dont ils définissent ce qu'ils voudraient que l'État incorpore à son système de lois - sont capables d'évoluer. Mais l'existence d'un forum de libre discussion est aussi la condition de cette recherche du bien commun. Celle-ci perd absolument toute signification lorsque ce forum disparaît. Le républicanisme est une foi dans les mérites de la confrontation libre entre les points de vue, et il demeure persuadé à la fois qu'il n'est pas vain de rechercher ce qui est bon en tant que cela est distinct de ce qui est intéressant pour moi, et que le forum public de discussion est la seule institution qui rende une telle recherche effectivement possible. En ce sens, le républicanisme reste à l'écart de toute philosophie politique qui prétend construire l'ordre institutionnel à partir de l'idée que les valeurs des individus sont à la fois ultimes, subjectives et inconciliables.

La deuxième conclusion est que la politique, prise au sens propre du terme, n'a rien à voir avec l'exercice du pouvoir; tout au contraire, elle est un effort pour supprimer les relations de pouvoir entre les hommes, et pour les remplacer par le règne d'une loi qui n'est pas l'institutionnalisation des rapports de forces mais qui, représentant l'intérêt commun, a cessé d'être un moyen de domination d'un homme sur un autre pour devenir un moyen d'union entre les hommes.

La troisième conclusion est qu'il n'y a rien de plus opposé à la république que la dictature d'une opinion qui prétendrait se former avant d'avoir été argumentativement confrontée, par des canaux institutionnels appropriés, à l'opinion contraire. Une république n'est pas un lieu où l'on enregistre ce que les gens pensent et veulent pour compter ensuite quel est le parti le plus nombreux ; c'est au contraire un lieu où l'on tente de mettre les différentes opinions en présence les unes des autres, et où on les oblige à se dépouiller de leur caractère particulier et « factionnel » en les contrai-

42. Machiavel, op. cit. supra n. 15, III, 28. 
gnant à délibérer du bien commun avec leurs rivales. La république n'est pas le calcul de la particularité la plus nombreuse, mais une technique pour l'élimination des particularismes.

La quatrième conclusion est que si le libertarianisme est une doctrine pernicieuse, le communautarisme l'est encore plus, et cela sous deux formes distinctes : la première serait l'idée que l'État doit organiser la coexistence entre des communautés qui demeurent unies autour de leurs intérêts et de leurs valeurs particulières et « factionnelles » sans accepter de les voir évoluer dans le contexte public de la discussion sur les règles qui doivent régir la vie collective; il ne serait donc qu'une instance d'arbitrage qui permettrait à des communautés de vivre ensemble dans une sorte de paix armée où chacune conserverait ses valeurs et son individualité. Contre cette forme de communautarisme, la république se présente comme un instrument de suppression des particularismes communautaires, et non comme l'outil de leur préservation. La seconde forme de communautarisme, c'est l'idée qu'une société ne peut pas survivre sans une certaine forme d'homogénéité dans les valeurs et dans les intérêts; il faudrait que les différents membres d'une communauté politique partagent les mêmes intérêts, les mêmes représentations du passé, aient les mêmes croyances religieuses, faute de quoi l'union entre les hommes deviendrait impossible. Contre cela, le républicanisme fait valoir que si les valeurs communes peuvent être un bien, ce n'est pas leur communauté qui en fait un bien mais leur validité; cela signifie qu'elles ne peuvent être des biens que si elles sont le produit d'une libre discussion : on progresse - ou on tente de progresser - vers la définition de ce qui est notre intérêt à tous, et vers la définition de règles de vie collective qui nous paraissent à la fois décentes et satisfaisantes. Mais aucun décret ne peut imposer ces valeurs communes, aucun obscurantisme qui ferait appel à l'enracinement dans la terre, dans l'histoire, dans la religion, dans la langue, etc. Par conséquent, si la seule manière d'avoir des valeurs communes est d'avoir un libre forum de discussion, on doit dire que, provisoirement et pour très longtemps, la seule valeur commune à laquelle les citoyens d'une libre république doivent demeurer attachés, c'est l'existence d'institutions libres permettant de rechercher ces valeurs communes et de progresser vers elles par l'échange et la confrontation des idées et des points de vue.

La cinquième conclusion est qu'au moment où la complexité du gouvernement des hommes s'accroit sans mesure, tous les arguments paraissent bons pour soustraire les détenteurs du pouvoir au contrôle de leurs mandants, et pour vider le principe démocratique de sa substance par la création d'une classe de politiciens professionnels qui sont pour ainsi dire inamovibles, et qui équilibrent de leur compétence et de leurs intérêts propres les désirs à courte vue des peuples qu'ils gouvernent. Cette struc- 
ture, censée tempérer les risques de démagogie et de populisme par l'existence d'une élite politique professionnelle, est précisément l'argumentaire contre lequel le républicanisme moderne s'est construit pour montrer que la démocratie pouvait être illimitée sans que cela implique pour autant le règne des démagogues; on voit d'ailleurs aujourd'hui que ceux-ci arrivent au contraire sur les talons de la professionnalisation excessive de la classe politique et de son autonomisation progressive par rapport à la volonté populaire. Ils ne sont pas le fruit d'une démocratie excessive mais bien d'un manque de démocratie.

Enfin, la dernière conclusion est que, depuis l'Antiquité, le principe démocratique de la souveraineté sans partage du peuple sur lui-même souffre d'un handicap théorique évident : il est accusé de promouvoir le règne des factions et de la démagogie, l'anarchie, l'injustice, le mépris des droits individuels et, pour finir, la tyrannie de la majorité qui débouche sur la tyrannie d'un seul. La théorie républicaine enseigne les moyens à mettre en ceuvre pour vaincre ce charme et sauver la démocratie de ses propres démons $^{43}$; elle est aussi une manière d'enseigner à la démocratie comment elle doit se comporter pour survivre durablement sans succomber aux maladies mortelles qui la guettent.

Jean-Fabien SPITZ (décembre 1994).

43. Cf. Drew R. McCoy, The Last of the fathers. James Madison and the republican legacy, Cambridge, Cambridge University Press, 1989, p. 58, 88, 128; cf., également, Stephen Holmes, Passions and constraints. On the theory of liberal democracy, Chicago, Chicago University Press, 1995, p. 29, 272; tout le livre de Holmes est consacré à l'idée que le pouvoir libéral et démocratique de l'époque moderne ne doit sa réalité qu'à sa propre limitation; c'est donc parce que les passions y sont contenues que la démocratie peut être un régime viable. 